not impede any research activities due to interference from railway operations or construction works," says Savill. Transport for London (TfL), the local government organization that is driving the Crossrail 2 plans, says that it takes the concerns "very seriously". The MRC is now working with TfL to find a solution.

The Crick team would like Crossrail 2 to be diverted back to its former route, away from the institute. But Michèle Dix, managing director for Crossrail 2, says that plans have already changed because of concerns from the Crick. Among other things, the planned tunnel has been shifted farther underground. "You can't just keep on moving it deeper and deeper," she says, adding that further concerns should be addressed through engineering - for example by making the tunnel linings thicker.

Daniel Moylan, a TfL board member and a transport adviser to London mayor Boris Johnson, says that the mayor is a huge supporter of both Crossrail 2 and the Crick. The latter is a major element in Johnson's plans to promote the capital as 'MedCity' - a global hub for life-sciences research. Moylan is confident that the institute's "legitimate" concerns can be allayed by means of technical solutions.

Objections from Parisian academics in the first half of the twentieth century are said to have affected the route of the Métro, but there have also been more-modern conflicts between scientific equipment and transport infrastructure.

\section{SCIENCE IN THE CITY}

The design of the Francis Crick Institute took into account existing railway lines, but not the latest proposed route for the Crossrail 2 line.

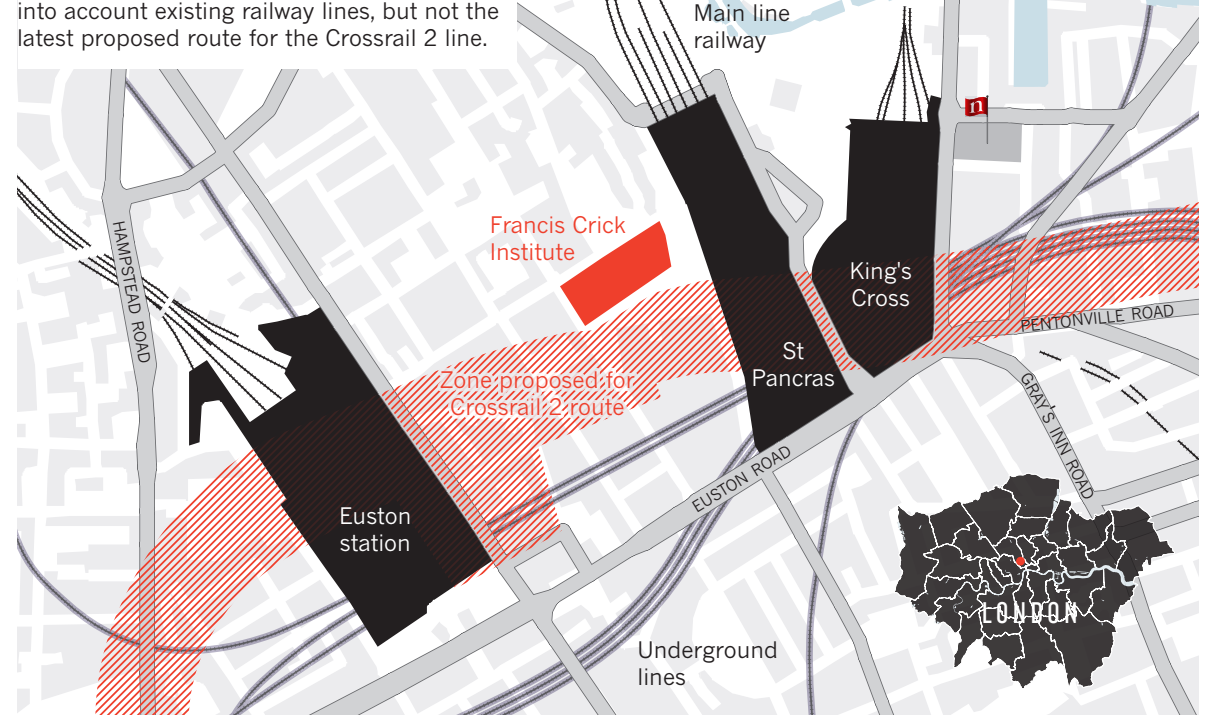

In 2013, the proposed route of a US light-rail line was diverted after concerns from the University of Colorado Denver about the effects on spectroscopy and microscopes at its medical campus in Aurora. In 2011, the University of Maryland in College Park dropped its opposition to a new 'Purple Line' link to the Washington DC Metro once the local transportation agency agreed to bury and shield power lines.
Other labs have depended on engineering solutions. The New York Structural Biology Center, located near a number of subway lines, placed its most sensitive equipment on concrete slabs attached directly to Manhattan bedrock. It experiences no problems from vibration now, says executive director Willa Appel. If there is no solution in London for the Crick, Appel says, "tell them they're welcome to come here".

\section{BY ELIZABETH GIBNEY}

$\mathrm{D}$ eepMind, the Google-owned artificialintelligence company, has revealed how it created a single computer algorithm that can learn how to play 49 different arcade games, including the 1970s classics Pong and Space Invaders. In more than half of those games, the computer became skilled enough to beat a professional human player.

The algorithm - which has generated a buzz since publication of a preliminary version in 2013 (V. Mnih et al. Preprint at http://arxiv.org/ $\mathrm{abs} / 1312.5602 ; 2013)$ - is the first artificialintelligence (AI) system that can learn a variety of tasks from scratch given only the same, minimal starting information. "The fact that you have one system that can learn several games,

without any tweaking from game to game, is surprising and pretty impressive," says Nathan Sprague, a machine-learning scientist at James Madison University in Harrisonburg, Virginia.

DeepMind, which is based in London, says that the brain-inspired system could also provide insights into human intelligence. "Neuroscientists are studying intelligence and decision-making, and here's a very clean test bed for those ideas," says Demis Hassabis, co-founder of DeepMind. He and his colleagues describe the gaming algorithm in a paper pub-

\section{ONATURE.COM}

For a video that

peeks inside the offices of DeepMind: go.nature.com/2kqata lished this week (V. Mnih et al. Nature 518, 529533 (2015); see also News \& Views on page 486).

Games are to AI researchers what fruit flies are to biology — a stripped-back system in which to test theories, says Richard Sutton, a computer scientist who studies reinforcement learning at the University of Alberta in Edmonton, Canada. "Understanding the mind is an incredibly difficult problem, but games allow you to break it down into parts that you can study," he says. But so far, most humanbeating computers - such as IBM's Deep Blue, which beat chess world champion Garry Kasparov in 1997, and the recently unveiled algorithm that plays Texas Hold 'Em poker essentially perfectly (see Nature http://doi. org/2dw; 2015) - excel at only one game.

DeepMind's versatility comes from joining two types of machine learning - an achievement that Sutton calls "a big deal". The first, called deep learning, uses a brain-inspired 